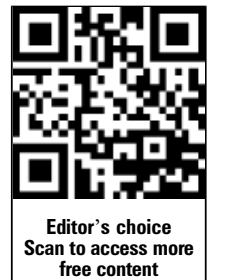

- Additional material is published online only. To view please visit the journal online (http://dx.doi.org/10.1136/ thoraxjnl-2012-202698).

For numbered affiliations see end of article.

\section{Correspondence to} Dr Guy G Brusselle, Department of Respiratory Medicine, Ghent University Hospital, De Pintelaan 185, Ghent B-9000, Belgium; guy.brusselle@ugent.be

Received 7 September 2012 Revised 13 November 2012 Accepted 26 November 2012 Published Online First 3 January 2013

\section{SLinked}

- http://dx.doi.org/10.1136/ thoraxjnl-2012-203055

- http://dx.doi.org/10.1136/ thoraxjnl-2013-203256

\title{
Azithromycin for prevention of exacerbations in severe asthma (AZISAST): a multicentre randomised double-blind placebo-controlled trial
}

\author{
Guy G Brusselle, ${ }^{1}$ Christine VanderStichele, ${ }^{1}$ Paul Jordens, ${ }^{2}$ René Deman, ${ }^{3}$ \\ Hans Slabbynck, ${ }^{4}$ Veerle Ringoet, ${ }_{1}^{5}$ Geert Verleden, ${ }^{6}$ Ingel K Demedts, ${ }^{7}$ \\ Katia Verhamme, ${ }^{8}$ Anja Delporte, ${ }^{1}$ Bénédicte Demeyere, ${ }^{1}$ Geert Claeys, ${ }^{9}$ \\ Jerina Boelens, ${ }^{9}$ Elizaveta Padalko, ${ }^{9}$ Johny Verschakelen, ${ }^{10}$ Georges Van Maele, ${ }^{11}$ \\ Ellen Deschepper, ${ }^{11}$ Guy F P Joos ${ }^{1}$
}

\section{ABSTRACT}

Background Patients with severe asthma are at increased risk of exacerbations and lower respiratory tract infections (LRTI). Severe asthma is heterogeneous, encompassing eosinophilic and non-eosinophilic (mainly neutrophilic) phenotypes. Patients with neutropilic airway diseases may benefit from macrolides.

Methods We performed a randomised double-blind placebo-controlled trial in subjects with exacerbationprone severe asthma. Subjects received low-dose azithromycin $(n=55)$ or placebo $(n=54)$ as add-on treatment to combination therapy of inhaled corticosteroids and long-acting $\beta_{2}$ agonists for 6 months. The primary outcome was the rate of severe exacerbations and LRTI requiring treatment with antibiotics during the 26-week treatment phase. Secondary efficacy outcomes included lung function and scores on the Asthma Control Questionnaire (ACQ) and Asthma Quality of Life Questionnaire (AQLQ).

Results The rate of primary endpoints (PEPS) during 6 months was not significantly different between the two treatment groups: 0.75 PEPs ( $95 \% \mathrm{Cl} 0.55$ to 1.01$)$ per subject in the azithromycin group versus $0.81 \mathrm{PEPs}(95 \% \mathrm{Cl}$ 0.61 to 1.09 ) in the placebo group ( $p=0.682$ ). In a predefined subgroup analysis according to the inflammatory phenotype, azithromycin was associated with a significantly lower PEP rate than placebo in subjects with noneosinophilic severe asthma (blood eosinophilia $\leq 200 / \mu \mathrm{l}$ ): 0.44 PEPs ( $95 \%$ Cl 0.25 to 0.78 ) versus 1.03 PEPs $(95 \%$ $\mathrm{Cl} 0.72$ to 1.48$)(p=0.013)$. Azithromycin significantly improved the AQLQ score but there were no significant between-group differences in the ACQ score or lung function. Azithromycin was well tolerated, but was associated with increased oropharyngeal carriage of macrolide-resistant streptococci.

Conclusions Azithromycin did not reduce the rate of severe exacerbations and LRTI in patients with severe asthma. However, the significant reduction in the PEP rate in azithromycin-treated patients with non-eosinophilic severe asthma warrants further study.

ClinicalTrials.gov number NCT00760838.

\section{INTRODUCTION}

Severe asthma is associated with substantial morbidity, disability and healthcare costs. ${ }^{12}$ In comparison

\section{Key messages}

What is the key question?

- Does maintenance treatment with low-dose azithromycin decrease the rate of exacerbations in adult patients with severe asthma and frequent exacerbations?

What is the bottom line?

- Azithromycin is a new option for prevention of exacerbations in patients with non-eosinophilic severe asthma.

Why read on?

- Severe asthma is a heterogeneous disease requiring different add-on treatments according to the underlying asthma phenotype (eosinophilic vs non-eosinophilic (eg, neutrophilic) airway inflammation).

with patients with mild-to-moderate asthma, adult patients with severe asthma have a higher need for medications, have more persistent symptoms and impaired lung function. Importantly, subjects with severe asthma have a greater frequency and severity of exacerbations of asthma, which puts them at risk of emergency department visits and hospitalisations. ${ }^{3}$ Moreover, severe asthma has been shown to be a risk factor for lower respiratory tract infections (LRTI), including pneumonia. ${ }^{4}$

Asthma is characterised by clinical and biological heterogeneity. ${ }^{4}$ Besides the well-known allergic eosinophilic asthma phenotype, half of patients with mild-to-moderate asthma have persistently non-eosinophilic disease. ${ }^{5}$ Interestingly, peripheral blood eosinophil counts correlate well with sputum eosinophilia, and a threshold of 220 eosinophils/ $\mu$ l blood was the best biomarker of sputum eosinophilia. ${ }^{5}$ Several phenotypes of severe asthma have been discerned by the Severe Asthma Research Program, demonstrating substantial differences in eosinophil and neutrophil counts in sputum. ${ }^{4}$ The non-eosinophilic asthma phenotype responds poorly to currently available anti-inflammatory 
therapy. ${ }^{5}{ }^{6}$ Subjects with severe asthma are older with longer disease duration, have less atopy by skin tests and frequently need oral corticosteroid courses despite multiple controller medications including high doses of inhaled corticosteroids. ${ }^{4}$ Relative corticosteroid insensitivity has indeed been implicated in patients with severe asthma and in smokers with asthma. ${ }^{7}$

Macrolides have immunomodulatory and anti-inflammatory effects in addition to their antibacterial effects. ${ }^{8}$ Maintenance treatment with macrolides such as azithromycin has been proved to be effective in chronic neutrophilic airway diseases including cystic fibrosis, bronchiectasis and diffuse panbronchiolitis. ${ }^{9-12}$ In an observational study, we have demonstrated the benefits of short-term macrolide treatment in patients with severe asthma. ${ }^{13}$ Recently, erythromycin and azithromycin—added to usual therapy-have been shown to prevent exacerbations in patients with chronic obstructive pulmonary disease (COPD), a predominantly neutrophilic airway disease. ${ }^{14-16}$

We conducted a randomised double-blind placebo-controlled trial to test the hypothesis that long-term add-on treatment with azithromycin decreases the frequency of acute exacerbations and LRTI in patients with exacerbation-prone severe asthma. Since severe asthma is a heterogeneous syndrome, we predefined to analyse the efficacy of azithromycin according to the type of underlying inflammation (non-eosinophilic (mainly neutrophilic) or eosinophilic asthma).

\section{METHODS}

\section{Study patients}

Patients were considered eligible if they were 18-75 years of age, had a diagnosis of persistent asthma, a history consistent with Global Initiative for Asthma step 4 or 5 clinical features, received high doses of inhaled corticosteroids $(\geq 1000 \mu \mathrm{g}$ fluticasone or equivalent) plus inhaled long-acting $\beta_{2}$ agonists for at least 6 months prior to screening and had had at least two independent severe asthma exacerbations requiring systemic corticosteroids and/or LRTI requiring antibiotics within the previous 12 months. Subjects were never-smokers or ex-smokers with a smoking history of $\leq 10$ pack-years. Their fractional excretion of exhaled nitric oxide ( $\mathrm{FeNO}$ ) level was below the upper limit of normal. Exclusion criteria are specified in the online supplementary appendix. Patients continued their maintenance treatment with high doses of inhaled corticosteroids and long-acting $\beta_{2}$ agonists during the trial.

\section{Study design and oversight}

The AZIthromycin in Severe ASThma (AZISAST) study was a randomised double-blind placebo-controlled parallel-group multicentre study (see online supplementary appendix figure S1). The study protocol was approved by the central ethics committee of Ghent University Hospital, and was reviewed by the local ethics committees at each participating site. All patients provided written informed consent.

\section{Randomisation and masking}

After a 2-week run-in period, patients were randomly assigned in a $1: 1$ ratio to receive add-on treatment with azithromycin or placebo using a central web-based randomisation tool. The hospital pharmacist (Ghent University Hospital) formulated the study drugs: capsules with $250 \mathrm{mg}$ azithromycin (prepared from capsules of Zitromax) or placebo. After randomisation, the patients took one capsule per day for 5 days and then one capsule three times a week. The total treatment period was 26 weeks (until visit 6), with a study drug-free follow-up period of 4 weeks (washout period).

\section{Assessments}

Assessments included asthma and medical history, vital signs, physical examination, electrocardiography, imaging, pulmonary function tests, FeNO measurements, blood testing and questionnaires (including the Asthma Control Questionnaire (ACQ) and the Asthma Quality of Life Questionnaire (AQLQ)). A full description of the assessments is given in the online supplementary appendix.

\section{Outcomes}

The primary efficacy outcome was the rate of primary endpoints (severe asthma exacerbations and/or LRTI requiring antibiotics) during the 26-week treatment phase. Severe asthma exacerbations were defined as deterioration in asthma leading to at least one of the following: (1) hospitalisation; (2) emergency room visit; and/or (3) need for systemic corticosteroids for at least 3 days. ${ }^{17}$

Secondary efficacy outcomes included lung function (forced expiratory volume in $1 \mathrm{~s}\left(\mathrm{FEV}_{1}\right)$ pre- and post-bronchodilation), morning and evening peak expiratory flow (PEF), quality of life (AQLQ score) and asthma control (ACQ score). All secondary outcomes were ascertained at visits 2, 3, 4, 5 and 6 (at randomisation and weeks $4,10,18$ and 26 of the treatment period), except for the questionnaires which were completed by the patient at visits 2, 4 and 6 only. Safety endpoints encompassed adverse events, serious adverse events and adverse events leading to discontinuation.

\section{Statistical analysis}

The primary outcome analysis was conducted within the intention-to-treat population. Unpaired and paired $t$ tests were used to assess between- and within-study group differences in symmetrically distributed continuous baseline characteristics and post-treatment outcome measures, respectively. Exact Wilcoxon rank-sum and signed rank tests were used for skewed distributed variables. Proportions were compared between both treatment groups using Fisher exact tests.

Mean primary endpoint rates and mean exacerbation rates per treatment group were investigated using Poisson or negative binomial regression as appropriate. ${ }^{18}$ For the first primary endpoint, log rank tests were performed and Kaplan-Meier curves are shown to present the cumulative survival in the placebo and azithromycin arms.

Receiver operating characteristic curve analysis was performed to assess the predictive power of the covariates. Statistical analyses were performed using IBM SPSS statistics V.19 (SPSS Inc, Chicago, Illinois, USA) and R V.2.14.1 (R Foundation for Statistical Computing, Vienna, Austria).

\section{RESULTS}

\section{Enrolment and baseline characteristics}

The flowchart of the AZISAST study is shown in figure 1 . A total of 109 of the 120 subjects screened were randomised and constituted the intention-to-treat population. Fifty-five subjects were randomly assigned to receive azithromycin and 54 subjects to receive placebo. Overall, 97\% of treatment visits were completed. Seven subjects who withdrew (two in the azithromycin group and five in the placebo group) completed a mean of four visits. Subjects in the two treatment arms were well matched with respect to baseline characteristics (table 1). All patients received high-dose combination therapy of inhaled corticosteroids and long-acting $\beta_{2}$ agonists for at least 6 months 


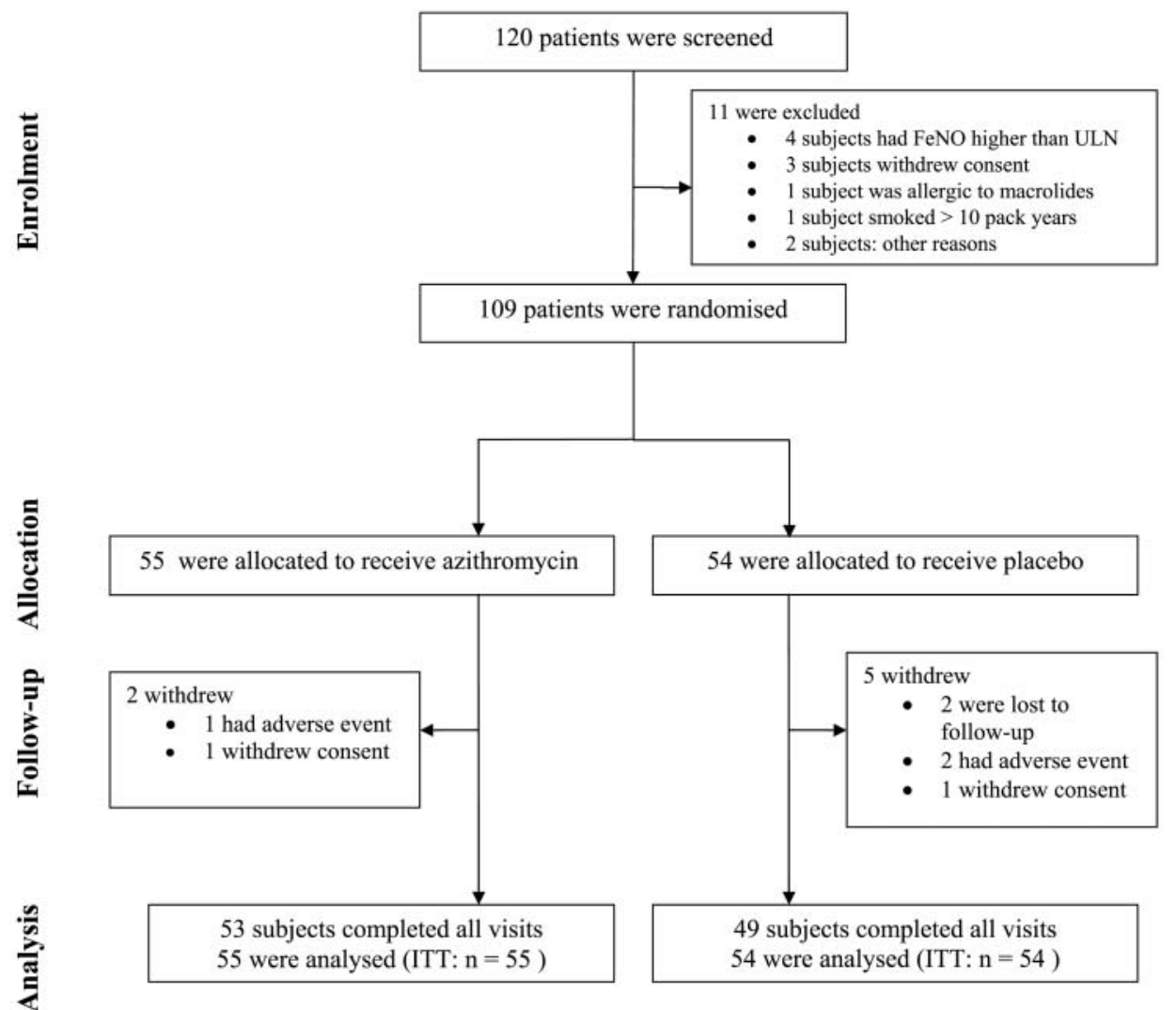

Figure 1 Numbers of patients who were enrolled, assigned to a study group and completed the study. Subjects were recruited by respiratory physicians at the seven participating clinical centres. Patients who completed the 26 -week course of the study drug were asked to return 4 weeks later for a washout visit. ULN, upper limit of normal; ITT, intention-to-treat.

prior to study entry and continued this treatment throughout the entire study.

\section{Efficacy}

Primary outcome

The median treatment period was 183 days in both the azithromycin group and the placebo group $(p=0.269)$. During this period a total of 39 primary endpoints (mean rate 0.72 per 26 weeks) occurred in the azithromycin group and 43 primary endpoints (mean rate 0.81 per 26 weeks) in the placebo group $(p=0.698)$. In the azithromycin group, $26(47 \%)$ subjects had at least one primary endpoint compared with 26 (48\%) in the placebo group (relative risk 0.98, 95\% CI 0.68 to 1.43, $\mathrm{p}=1.000$ ). The cumulative survival times based on the first primary endpoint per patient are shown as Kaplan-Meier survival curves for both treatment arms in figure $2 \mathrm{~A}(\mathrm{p}=0.801)$. The number of primary endpoints per patient is shown in figure 2B $(\mathrm{p}=0.698)$.

Thirty severe exacerbations of asthma occurred in the azithromycin group compared with 27 in the placebo group $(p=1.000)$. Twenty patients in the azithromycin group and 29 patients in the placebo group experienced a LRTI requiring antibiotics $(p=0.826)$. There were two hospital admissions for exacerbations of asthma in the azithromycin group and two in the placebo group $(p=1.000)$.

The estimated primary endpoint rate based on a Poisson regression model without adjustment was 0.71 (95\% CI 0.52 to 0.97 ) in the azithromycin group and 0.80 (95\% CI 0.59 to
1.07 ) in the placebo group (estimated primary endpoint rate ratio for azithromycin vs placebo $0.89,95 \%$ CI 0.58 to 1.37 , $\mathrm{p}=0.600)$. After imputation, the estimated adjusted primary endpoint rate during 6 months was 0.75 (95\% CI 0.55 to 1.01) in the azithromycin group and 0.81 (95\% CI 0.61 to 1.09 ) in the placebo group (estimated rate ratio $0.92,95 \%$ CI 0.60 to $1.40, \mathrm{p}=0.682$ ). A negative binomial regression model did not alter the results. ${ }^{18}$ When sensitivity analyses restricting the primary endpoint to severe exacerbations of asthma were performed, the estimated severe exacerbation rate based on a Poisson regression model was 0.55 (95\% CI 0.38 to 0.78 ) in the azithromycin group and $0.52(95 \%$ CI 0.36 to 0.75$)$ in the placebo group (estimated primary endpoint rate ratio for azithromycin vs placebo $1.05,95 \%$ CI 0.63 to $1.76, \mathrm{p}=0.847$ ).

Predefined subgroup analyses

Since severe asthma is biologically heterogeneous, we performed a predefined subgroup analysis comparing the efficacy of azithromycin depending on blood eosinophilia at baseline. In subjects with severe asthma and blood eosinophilia $\leq 200 / \mu \mathrm{l}$ (non-eosinophilic severe asthma), azithromycin significantly reduced the rate of primary endpoints and of severe exacerbations compared with placebo (figure 2C). The estimated primary endpoint rate for non-eosinophilic severe asthma was 0.44 (95\% CI 0.25 to 0.78 ) in the azithromycin group and 1.03 (95\% CI 0.72 to 1.48 ) in the placebo group (estimated primary endpoint rate ratio for azithromycin vs placebo $0.43,95 \% \mathrm{CI}$ 0.22 to $0.84, p=0.013$ ). The estimated severe exacerbation rate 
Table 1 Baseline characteristics of subjects in the intention-to-treat population

\begin{tabular}{|c|c|c|c|}
\hline Characteristic & Placebo $(\mathrm{N}=54)$ & Azithromycin (N=55) & $\mathrm{p}$ Value \\
\hline \multicolumn{4}{|l|}{ Sex, $n$} \\
\hline Male & $16(30 \%)$ & $26(47 \%)$ & \multirow[t]{2}{*}{0.077} \\
\hline Female & $38(70 \%)$ & $29(53 \%)$ & \\
\hline \multicolumn{4}{|l|}{ Age, years } \\
\hline Median (range), IQR & $53(20-74),(36-60)$ & 53 (19-76), (46-64) & 0.097 \\
\hline \multicolumn{4}{|l|}{ Age at onset of symptoms, years } \\
\hline Median (range), IQR & $17(1-72),(6-38)$ & $20(0-71),(3-40)$ & 0.828 \\
\hline \multicolumn{4}{|l|}{ Asthma duration, years } \\
\hline Median (range) IQR & $23(1-63),(12.8-41.3)$ & $27(2-70),(11-45)$ & 0.263 \\
\hline \multicolumn{4}{|l|}{ Race, $n(\%)$ of subjects } \\
\hline Caucasian & $54(100 \%)$ & $55(100 \%)$ & - \\
\hline \multicolumn{4}{|l|}{ Body mass index* } \\
\hline Mean (SD) & $26.4(5.4)$ & $26.5(4.9)$ & 0.926 \\
\hline Positive atopic status, $\mathrm{n}(\%)$ of subjects $†$ & $38(70 \%)$ & $35(64 \%)$ & 0.542 \\
\hline \multicolumn{4}{|l|}{ Total lgE (IU/ml) } \\
\hline Median (range), IQR & $87.3(2-4500),(25.2-702.7)$ & $111.3(1-5000),(30.4-266.0)$ & 0.685 \\
\hline History of nasal polyps, $n(\%)$ of subjects & $6(11 \%)$ & $11(20 \%)$ & 0.291 \\
\hline Hospitalisations due to asthma in previous year, $\mathrm{n}(\%)$ of subjects & $13(24 \%)$ & $13(24 \%)$ & 1.000 \\
\hline Emergency room visits due to asthma in previous year, $\mathrm{n}(\%)$ of subjects & $8(15 \%)$ & $4(7 \%)$ & 0.237 \\
\hline Severe asthma exacerbations requiring OCS in previous year, $\mathrm{n}(\%)$ of subjects & $47(87 \%)$ & $49(89 \%)$ & 0.776 \\
\hline LRTI requiring antibiotics in previous year, $n(\%)$ of subjects & $44(82 \%)$ & $46(84 \%)$ & 0.805 \\
\hline \multicolumn{4}{|l|}{ Severe asthma exacerbations and/or LRTI requiring antibiotics in previous year } \\
\hline $\mathrm{N}$ (mean) & $3.0(1.28)$ & $3.4(2.08)$ & 0.536 \\
\hline \multicolumn{4}{|l|}{$\mathrm{FEV}_{1}$ prebronchodilator (\% of predicted) } \\
\hline Mean (SD) & $84.8(20.7)$ & $80.1(21.9)$ & 0.287 \\
\hline \multicolumn{4}{|l|}{$\mathrm{FEV}_{1} / \mathrm{FVC}$ ratio prebronchodilator } \\
\hline Mean (SD) & $67.8(12.1)$ & $66.8(12.3)$ & 0.556 \\
\hline \multicolumn{4}{|l|}{$\mathrm{FEV}_{1}$ postbronchodilator (\% of predicted) } \\
\hline Mean (SD) & $89.3(19.2)$ & $83.9(21.7)$ & 0.184 \\
\hline \multicolumn{4}{|l|}{ Improvement in $\mathrm{FEV}_{1}$ after $\mathrm{BD}$ use (\%) } \\
\hline Mean (SD) & $6.5(9.0)$ & $5.5(7.6)$ & 0.959 \\
\hline \multicolumn{4}{|l|}{ FeNO (ppb)‡ } \\
\hline Median (range), IQR & 17.5 (6-63), (12-27.5) & $18.0(4-54),(14-29)$ & 0.519 \\
\hline \multicolumn{4}{|l|}{ Eosinophil count in blood $\left(\times 10^{9} / \mathrm{l}\right)$} \\
\hline Median (range), IQR & $186(40-1200),(109-354)$ & $208(0-1240),(100-370)$ & 0.901 \\
\hline \multicolumn{4}{|l|}{ Score on ACQ-7 } \\
\hline Mean (SD) & $1.7(1.0)$ & $1.4(0.9)$ & 0.400 \\
\hline \multicolumn{4}{|l|}{ Score on AQLQ } \\
\hline Mean (SD) & $5.2(1.1)$ & $5.5(0.9)$ & 0.287 \\
\hline \multicolumn{4}{|l|}{ Daily dose of inhaled corticosteroid§ $(\mu \mathrm{g})$} \\
\hline Median (range) & $2000(1000-4000)$ & $2000(1000-4000)$ & 0.805 \\
\hline \multicolumn{4}{|l|}{ Regular use of oral prednisolone } \\
\hline $\mathrm{N}(\%)$ of subjects & $3(6 \%)$ & $9(16 \%)$ & 0.124 \\
\hline \multicolumn{4}{|l|}{ Daily maintenance dose (mg) } \\
\hline Median (range) & $10(2.5-17.5)$ & $10(2.5-10)$ & 0.359 \\
\hline \multicolumn{4}{|l|}{ Use of montelukast (LTRA) } \\
\hline $\mathrm{N}(\%)$ of subjects & $26(48 \%)$ & $29(53 \%)$ & 0.703 \\
\hline
\end{tabular}

*Body mass index is the weight in kilograms divided by the square of the height in metres.

†Atopic status based on skin prick tests; if skin prick test was not interpretable or not available, the atopic status is based on serum RAST for standard aeroallergens (house dust mite, animal dander (cat, dog), pollen (grass, tree) and Aspergillus fumigatus).

\#FeNO was measured at a flow rate of $50 \mathrm{ml} / \mathrm{s}$ and expressed as parts per billion (ppb).

$\S$ The doses of inhaled corticosteroids were converted to the equivalent dose of beclomethasone dipropionate and expressed as beclomethasone dipropionate equivalent.

ACQ, Asthma Control Questionnaire; AQLQ, Asthma Quality of Life Questionnaire; FeNO, fraction of exhaled nitric oxide; FEV 1 , forced expiratory volume in $1 \mathrm{~s}$; LRTI, lower respiratory

tract infection; LTRA, leukotriene receptor antagonist; OCS, oral corticosteroids.

for non-eosinophilic severe asthma was 0.26 (95\% CI 0.12 to $0.54)$ in the azithromycin group and 0.62 (95\% CI 0.39 to 0.99 ) in the placebo group (estimated severe exacerbation rate ratio for azithromycin vs placebo $0.42,95 \%$ CI 0.17 to 1.00 , $\mathrm{p}=0.050)$. In contrast, the primary endpoint rate for eosinophilic severe asthma was 0.96 (95\% CI 0.66 to 1.41$)$ in the azithromycin group compared with 0.50 (95\% CI 0.28 to 0.88 ) in the placebo group (estimated rate ratio $1.93,95 \% \mathrm{CI}$ 0.98 to $3.81, \mathrm{p}=0.058$ ). In patients with eosinophilic severe asthma, the severe exacerbation rate was higher in the 

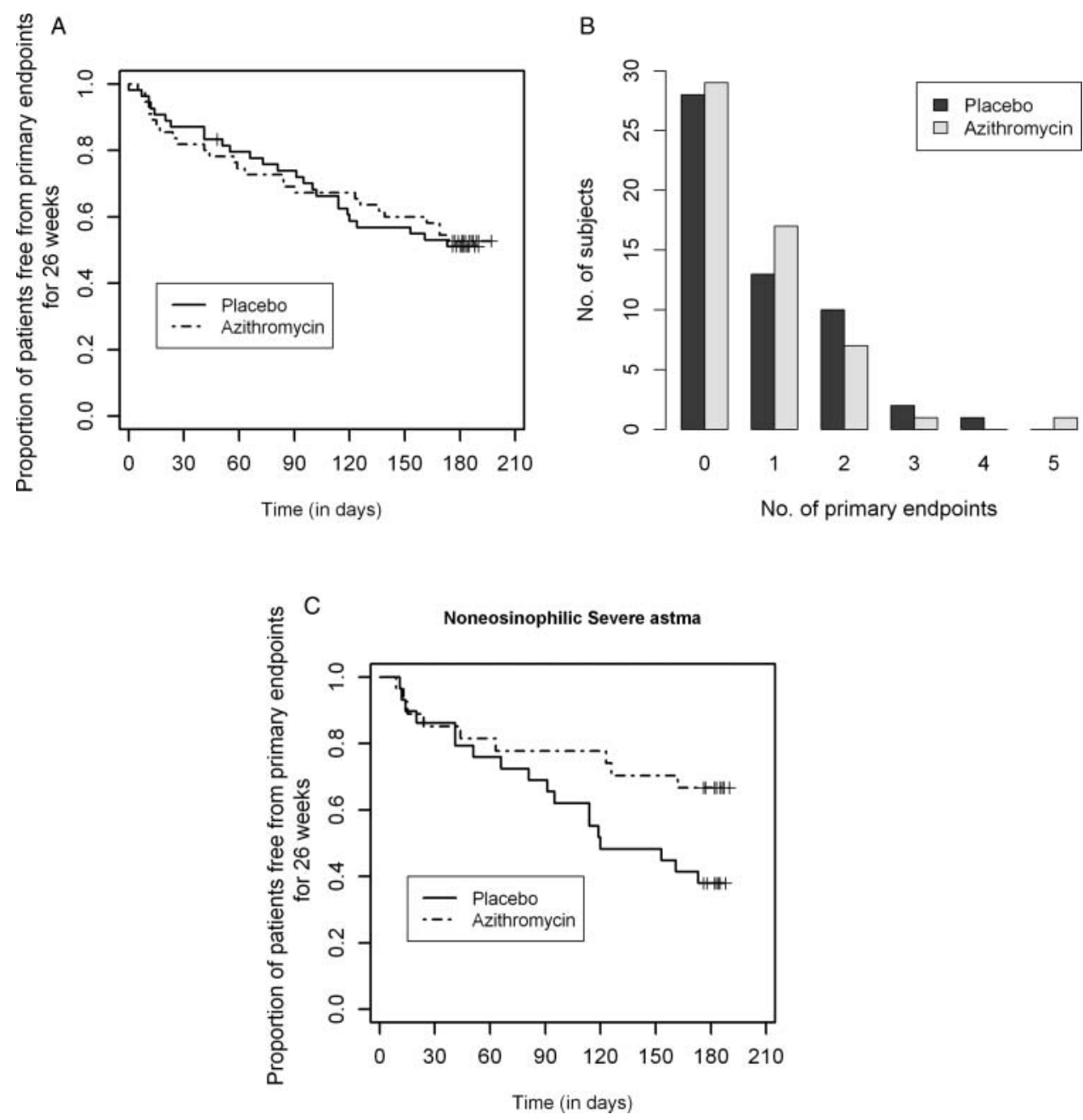

Figure 2 Primary endpoints during the course of the study. (A) Proportion of participants free from primary endpoints for 26 weeks according to study group. The intention-to-treat analyses were based on the participants who were randomly assigned to azithromycin ( $\mathrm{N}=55)$ or placebo ( $\mathrm{N}=54)$. A primary endpoint was defined as a severe asthma exacerbation requiring treatment with systemic corticosteroids, emergency room visit or hospitalisation ${ }^{17}$ and/or an acute lower respiratory tract infection requiring treatment with antibiotics. (B) Distribution of the number of primary endpoints among subjects in each study group during the treatment period of the study. (C) Proportion of subjects with non-eosinophilic severe asthma (defined by a fraction of exhaled nitric oxide lower than the upper limit of normal and a blood eosinophilia $\leq 200 / \mu$ l) free from primary endpoints for 26 weeks, according to study group (azithromycin or placebo). In subjects with non-eosinophilic asthma, azithromycin significantly decreased the number of patients with at least one primary endpoint (9 of 27 (33\%) azithromycin-treated subjects vs 18 of 29 (62\%) placebo-treated subjects; relative risk $0.54,95 \% \mathrm{Cl} 0.29$ to $0.98, \mathrm{p}=0.037)$.

azithromycin group than in the placebo group: $0.82(95 \% \mathrm{CI}$ 0.55 to 1.24 ) versus 0.38 (95\% CI 0.20 to 0.72 ) estimated rate ratio $2.19,95 \%$ CI 1.01 to $4.73, \mathrm{p}=0.046)$. In the Poisson regression model there is a significant interaction between the phenotype of severe asthma and treatment arm $(p=0.002)$.

Other efficacy outcomes

At 26 weeks there was a significant improvement in the AQLQ score in the azithromycin group $(0.32,95 \%$ CI 0.08 to 0.57 , $\mathrm{p}=0.011)$ compared with a non-significant trend in the placebo group $(0.20,95 \% \mathrm{CI}-0.01$ to $0.41, \mathrm{p}=0.057$; table 2$)$. There were no significant differences between the groups in the change from baseline in AQLQ score (mean difference 0.12; $95 \% \mathrm{CI}-0.20$ to $0.44 ; \mathrm{p}=0.467)$.

At 26 weeks, the mean improvement in the ACQ score was $-0.24(95 \% \mathrm{CI}-0.50$ to $0.02, \mathrm{p}=0.068)$ in the azithromycin group compared with $-0.12(95 \% \mathrm{CI}-0.33$ to $0.08, \mathrm{p}=0.222)$ in the placebo group (table 2). There were no significant between-group differences in the change from baseline in the
ACQ score (mean difference $-0.12 ; 95 \%$ CI -0.44 to 0.21 ; $\mathrm{p}=0.485)$. There were no significant between-group differences in the changes in $\mathrm{FEV}_{1}$ (pre- and post-bronchodilator), morning PEF, evening PEF, use of rescue medication and FeNO (table 2).

\section{Safety}

No significant differences were observed in the frequency of adverse events, serious adverse events or adverse events leading to discontinuation of the study drug (see online supplementary table S1). Importantly, no subject in the azithromycin group reported hearing loss.

\section{Oropharyngeal colonisation and resistance to macrolides}

Two clinical centres studied resistance to macrolide antibiotics, obtaining oropharyngeal swabs in 46 participants (23 in each treatment arm) at four visits (see online supplemental figure S1). Eleven subjects (47.8\%) in the azithromycin group and nine subjects $(39.1 \%)$ in the placebo group were colonised with erythromycin-resistant streptococci in the oropharynx at 


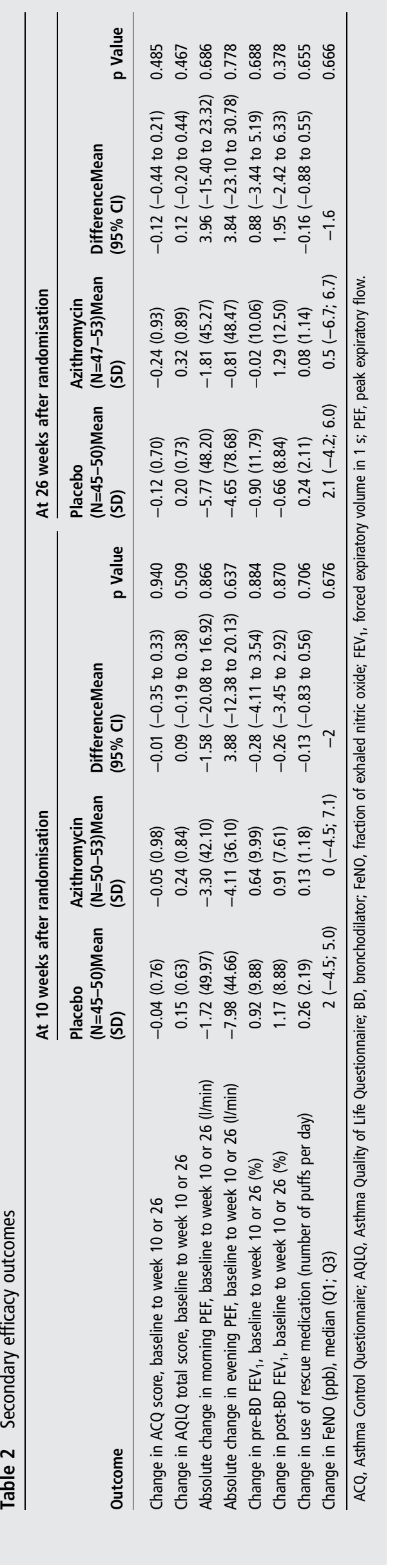

randomisation. At the end of the 26-week treatment period, $87 \%$ of the subjects in the azithromycin group and $35 \%$ of the subjects in the placebo group were colonised with erythromycinresistant oropharyngeal streptococci $(p<0.001)$. During the treatment period the proportion of streptococci resistant to erythromycin increased from $17.2 \%$ to $73.8 \%$ in the azithromycin group and from $7.9 \%$ to $17.3 \%$ in the placebo group $(p<0.001$; see online supplemental figure S3). The percentage of macrolide-resistant streptococci numerically decreased from $73.8 \%$ to $45.9 \%$ in the azithromycin group during the 4-week washout period $(\mathrm{p}=0.104)$.

\section{DISCUSSION}

In this randomised double-blind placebo-controlled trial in patients with severe asthma, add-on treatment with low-dose azithromycin for 6 months did not decrease the frequency of the primary endpoint (severe exacerbations of asthma and LRTI requiring antibiotics). However, in a predefined subgroup analysis-namely, in subjects with severe non-eosinophilic asthma (as defined by a FeNO lower than the upper limit of normal and a blood eosinophilia $\leq 200 / \mu$ l (the median value of blood eosinophilia in our ITT population))-add-on treatment with azithromycin was associated with a significant reduction in primary endpoints and in the rate of severe exacerbations. Azithromycin improved quality of life and was well tolerated.

Several studies have examined whether macrolides are beneficial in adult patients with asthma. ${ }^{19-23}$ However, interpretation of the studies is difficult because of the heterogeneous study populations, the small number of patients studied and the short study durations (less than 12 weeks). ${ }^{8}{ }^{24}$ Most studies have been performed in patients with mild-to-moderate asthma. ${ }^{19-21} 2325$ In a proof-of-concept study, Simpson and colleagues demonstrated that clarithromycin-as add-on treatment to inhaled corticosteroids for 8 weeks in patients with severe asthmasignificantly reduced airway concentrations of interleukin- 8 and neutrophil numbers and improved quality of life, especially in patients with refractory non-eosinophilic asthma. ${ }^{26}$ However, the duration of this single-centre study was too short and the number of patients too small to examine the effect of macrolides on exacerbations. Also, in our multicentre study, treatment with azithromycin significantly improved quality of life at 26 weeks compared with baseline. In both studies there were no changes in symptom scores, lung function or FeNO levels with macrolide treatment. ${ }^{26}$

Since severe asthma is a heterogeneous syndrome, we predefined to analyse the efficacy of azithromycin according to the asthma phenotype (non-eosinophilic (mainly neutrophilic) or eosinophilic asthma). ${ }^{1}{ }^{2}$ Add-on treatment with azithromycin significantly decreased the rate of primary endpoints and of severe exacerbations in the subgroup of patients with non-eosinophilic severe asthma. In contrast, in subjects with eosinophilic severe asthma, there was a trend towards an increased rate of primary endpoints in the azithromycin group, in line with case reports describing the induction of ChurgStrauss syndrome in patients with eosinophilic asthma receiving add-on treatment with azithromycin. ${ }^{27}$

The beneficial effects of azithromycin in non-eosinophilic severe asthma might be due to antibiotic properties or antiinflammatory and immunomodulatory effects. Chronic respiratory infection with atypical bacteria such as Mycoplasma pneumoniae or Chlamydophila pneumoniae might play a role in the pathogenesis of severe asthma. ${ }^{28}$ However, a trial of roxithromycin in subjects with asthma and serological evidence of infection with $C$ pneumoniae did not improve asthma control, ${ }^{20}$ 
which is in line with our observations that positive IgG antibodies to $C$ pneumoniae did not predict therapeutic efficacy of azithromycin in severe asthma.

Long-term treatment with azithromycin in our study appeared to be safe, since the frequency and severity of adverse events was not different from placebo. In particular, no subjects in the azithromycin-treated group mentioned hearing loss, which contrasts with the hearing decrements reported by Albert et al in patients with chronic obstructive pulmonary disease (COPD). ${ }^{15}$ The older age of the subjects with COPD, more frequent comorbidities, the higher dose of azithromycin used and the intensive monitoring by means of audiometry in the COPD study might explain this difference. Recently, a retrospective observational database study has suggested a small increased risk of cardiovascular death among patients with a high baseline risk of cardiovascular disease taking azithromycin during 5 days for acute infections. ${ }^{29}$ Since we excluded patients with significant cardiovascular disease, a prolonged corrected QT interval or use of drugs known to cause QT prolongation, there were no serious cardiac adverse drug reactions in our study.

A concern of chronic treatment with azithromycin is the induction of resistance to macrolides. Short-term treatment with macrolides induced a significant increase in macrolide-resistant pharyngeal streptococci in healthy volunteers. ${ }^{30}$ In our study, long-term treatment with azithromycin was associated with an increased proportion of macrolide-resistant oropharyngeal streptococci, confirming the increased incidence of macrolide resistance in the nasopharyngeal flora in the COPD Clinical Research Network study. ${ }^{15}$ However, in both studies, there is no evidence suggesting that colonisation with macrolide-resistant organisms increased the risk of LRTI or pneumonia.

Our study has several strengths, including the double-blind design, web-based randomisation and the concealment of allocation. The AZISAST study also has limitations, including the absence of induced sputum or bronchoscopy to delineate the underlying airway inflammation. However, to maximise the external validity of our study, we did not perform induced sputum examinations since this labour-intensive procedure is mainly performed in specialised tertiary referral centres. Moreover, peripheral blood eosinophilia is a sensitive and specific biomarker for airway eosinophilia, both after allergen challenge and in chronic asthma. ${ }^{5}$ Whereas phase II trials of targeted add-on therapies with the antiinterleukin 5 monoclonal antibody mepolizumab in refractory eosinophilic asthma initially requested increased eosinophil counts in sputum, ${ }^{31}$ an increased blood eosinophil count has been used as a qualifying inclusion criterion in the phase III trial of mepolizumab (DREAM study). ${ }^{32}$ Importantly, a FeNO level below the upper limit of normal was an inclusion criterion in our study to avoid enrolment of patients with exacerbation-prone severe asthma due to non-adherence to inhaled corticosteroids. A high FeNO level $(>50 \mathrm{ppb})$ in a symptomatic patient with an established diagnosis of asthma indeed implies deteriorating eosinophilic airway inflammation, most frequently due to poor adherence to inhaled corticosteroids. ${ }^{33}$

In summary, this is the first randomised controlled trial examining the efficacy and safety of add-on treatment with low-dose azithromycin in adults with exacerbation-prone severe asthma. Although azithromycin was not superior to placebo in the total population, we demonstrated a significant reduction in primary endpoints in non-eosinophilic severe asthma. This observation is biologically plausible, since macrolides have been shown to be effective in neutrophilic chronic airway diseases such as cystic fibrosis (CF), non-CF bronchiectasis, diffuse panbronchiolitis and COPD. The induction of macrolide resistance in the nasopharyngeal and oropharyngeal flora in azithromycin-treated subjects is of concern. In addition, the long-term effects of macrolide treatment on microbial resistance in the community are not known.

\section{Author affiliations}

${ }^{1}$ Department of Respiratory Medicine, Ghent University Hospital, Ghent, Belgium

${ }^{2}$ Department of Respiratory Medicine, OLV Ziekenhuis, Aalst, Belgium

${ }^{3}$ Department of Respiratory Medicine, AZ Groeninge, Kortrijk, Belgium

${ }^{4}$ Department of Respiratory Medicine, ZNA Middelheim, Antwerpen, Belgium

${ }^{5}$ Department of Respiratory Medicine, AZ Sint-Jan, Brugge, Belgium

${ }^{6}$ Department of Respiratory Medicine, University Hospital Gasthuisberg, Leuven, Belgium

${ }^{7}$ Department of Respiratory Medicine, Heilig Hart Ziekenhuis, Roeselare, Belgium ${ }^{8}$ Department of Epidemiology, Erasmus MC Rotterdam, Rotterdam, The Netherlands ${ }^{9}$ Department of Microbiology, Clinical Chemistry and Immunology, Ghent University Hospital, Ghent, Belgium

${ }^{10}$ Department of Radiology, University Hospital Gasthuisberg, Leuven, Belgium

${ }^{11}$ Biostatistics Unit, Department of Public Health, Ghent University Hospital, Ghent, Belgium

Acknowledgements We acknowledge all patients, physicians, clinical trial nurses, data managers and other collaborators who contributed to the AZISAST study.

Contributors GGB: study design. GGB, CVS, PJ, RD, HS, VR, GVM, IKD, GFPJ: patient recruitment, enrolment, randomisation and follow-up. $A D, B D$, JV: collection of the data. GC, JB and EP: bacteriological and serological analyses. GGB, CVS, KV, GVM, ED, GFPJ: analysis and interpretation of the data. GGB, GVM, KV, JB, EP, ED, GFPJ: writing of the report.

\section{Competing interests None.}

Patient consent All patients provided written informed consent, as required by Belgian Law and the Ethical Committees involved.

Ethics approval The study protocol was approved by the central ethics committee of Ghent University Hospital, and was reviewed by the local ethics committees at each participating site.

Funding The AZIthromycin in Severe ASThma (AZISAST) study was an academic clinical trial, without sponsorship from the pharmaceutical industry. The study was funded by the Agency for Innovation by Science and Technology (IWT 70709), Flanders, Belgium. The study sponsor (IWT) had no role in the study design; in the collection, analysis, and interpretation of data; in the writing of the report; and in the decision to submit the paper for publication.

Provenance and peer review Not commissioned; internally peer reviewed.

\section{REFERENCES}

1 Wenzel SE. Asthma: defining of the persistent adult phenotypes. Lancet 2006;368:804-13.

2 The ENFUMOSA cross-sectional European multicentre study of the clinical phenotype of chronic severe asthma. European Network for Understanding Mechanisms of Severe Asthma. Eur Respir J 2003;22:470-7.

3 Haselkorn T, Fish JE, Zeiger RS, et al. Consistently very poorly controlled asthma, as defined by the impairment domain of the Expert Panel Report 3 guidelines, increases risk for future severe asthma exacerbations in The Epidemiology and Natural History of Asthma: Outcomes and Treatment Regimens (TENOR) study. J Allergy Clin Immunol 2009;124:895-902 e1-4.

4 Moore WC, Meyers DA, Wenzel SE, et al. Identification of asthma phenotypes using cluster analysis in the Severe Asthma Research Program. Am J Respir Crit Care Med 2010;181:315-23.

5 McGrath KW, Icitovic N, Boushey HA, et al. A large subgroup of mild-to-moderate asthma is persistently noneosinophilic. Am J Respir Crit Care Med 2012;185:612-19.

6 Green RH, Brightling CE, Woltmann G, et al. Analysis of induced sputum in adults with asthma: identification of subgroup with isolated sputum neutrophilia and poor response to inhaled corticosteroids. Thorax 2002;57:875-9.

7 Thomson NC, Chaudhuri R, Livingston E. Asthma and cigarette smoking. Eur Respir J 2004;24:822-33.

8 Crosbie PAJ, Woodhead MA. Long-term macrolide therapy in chronic inflammatory airway diseases. Eur Respir J 2009;33:171-81.

9 Equi A, Balfour-Lynn IM, Bush A, et al. Long term azithromycin in children with cystic fibrosis: a randomised, placebo-controlled crossover trial. Lancet 2002;360:978-84.

10 Davies G, Wilson R. Prophylactic antibiotic treatment of bronchiectasis with azithromycin. Thorax 2004;59:540-1.

11 Wong C, Jayaram L, Karalus N, et al. Azithromycin for prevention of exacerbations in non-cystic fibrosis bronchiectasis (EMBRACE): a randomised, double-blind, placebo-controlled trial. Lancet 2012:380:660-7. 
12 Koyama H, Geddes DM. Erythromycin and diffuse panbronchiolitis. Thorax 1997;52:915-18.

13 Coeman M, van Durme Y, Bauters F, et al. Neomacrolides in the treatment of patients with severe asthma and/or bronchiectasis: a retrospective observational study. Ther Adv Respir Dis 2011;5:377-86.

14 Seemungal TA, Wilkinson TM, Hurst JR, et al. Long-term erythromycin therapy is associated with decreased chronic obstructive pulmonary disease exacerbations. Am J Respir Crit Care Med 2008;178:1139-47.

15 Albert RK, Connett J, Bailey WC, et al. Azithromycin for prevention of exacerbations of COPD. N Engl J Med 2011;365:689-98.

16 Brusselle GG, Joos GF, Bracke KR. New insights into the immunology of chronic obstructive pulmonary disease. Lancet 2011;378:1015-26.

17 Reddel HK, Taylor DR, Bateman ED, et al. An official American Thoracic Society/ European Respiratory Society statement: asthma control and exacerbations: standardizing endpoints for clinical asthma trials and clinical practice. Am J Respir Crit Care Med 2009;180:59-99.

18 Keene ON, Calverley PM, Jones PW, et al. Statistical analysis of COPD exacerbations. Eur Respir 」 2008;32:1421-2.

19 Kraft M, Cassell GH, Pak J, et al. Mycoplasma pneumoniae and Chlamydia pneumoniae in asthma: effect of clarithromycin. Chest 2002;121:1782-8.

20 Black PN, Blasi F, Jenkins CR, et al. Trial of roxithromycin in subjects with asthma and serological evidence of infection with Chlamydia pneumoniae. Am J Respir Crit Care Med 2001;164:536-41

21 Shoji T, Yoshida S, Sakamoto H, et al. Anti-inflammatory effect of roxithromycin in patients with aspirin-intolerant asthma. Clin Exp Allergy 1999;29:950-6.

22 Simpson JL, Powell H, Boyle MJ, et al. Clarithromycin targets neutrophilic airway inflammation in refractory asthma. Am J Respir Crit Care Med 2008;177:148-55.
23 Sutherland ER, King TS, Icitovic N, et al. A trial of clarithromycin for the treatment of suboptimally controlled asthma. J Allergy Clin Immunol 2010;126:747-53.

24 Richeldi L, Ferrara G, Fabbri LM, et al. Macrolides for chronic asthma. Cochrane Database Syst Rev 2005;(4):CD002997.

25 Amayasu H, Yoshida S, Ebana S, et al. Clarithromycin suppresses bronchial hyperresponsiveness associated with eosinophilic inflammation in patients with asthma. Ann Allergy Asthma Immunol 2000;84:594-8.

26 Simpson JL, Powell H, Boyle MJ, et al. Clarithromycin targets neutrophilic airway inflammation in refractory asthma. Am J Respir Crit Care Med 2008;177:148-55.

27 Hübner C, Dietz A, Stremmel W, et al. Macrolide-induced Churg-Strauss syndrome in a patient with atopy. Lancet 1997;350:563.

28 Martin RJ, Kraft M, Chu HW, et al. A link between chronic asthma and chronic infection. J Allergy Clin Immunol 2001;107:595-601.

29 Ray WA, Murray KT, Hall K, et al. Azithromycin and the risk of cardiovascular death N Engl J Med 2012;366:1881-90.

30 Malhotra-Kumar S, Lammens C, Coenen S, et al. Effect of azithromycin and clarithromycin therapy on pharyngeal carriage of macrolide-resistant streptococci in healthy volunteers: a randomised, double-blind, placebo-controlled study. Lancet 2007;369:482-90.

31 Haldar P, Brightling CE, Hargadon B, et al. Mepolizumab and exacerbations of refractory eosinophilic asthma. N Engl J Med 2009;360:973-84.

32 Pavord ID, Korn S, Howarth P, et al. Mepolizumab for severe eosinophilic asthma (DREAM): a multicentre, double-blind, placebo-controlled trial. Lancet 2012;380:651-9.

33 Dweik RA, Boggs PB, Erzurum SC, et al. An official ATS clinical practice guideline: interpretation of exhaled nitric oxide levels (FENO) for clinical applications. Am J Respir Crit Care Med 2011;184:602-15. 